\title{
Nucleus penetrating peptidomimetic synthesis and application
}

\author{
Magdalena Wysocka, Anita Romanowska, Irena Audzeyenka, Adam Lesner
}

University of Gdansk, Poland

https://doi.org/10.17952/35EPS.2018.158

Arginine rich peptides belongs to class of cell penetrating peptides (CPPs) that can transport the wide variety of attached chemical entities (cargoes) through the cell membrane. Diversity of transported molecules is impressive and includes DNAs, siRNAs, peptides, proteins and small drugs. Polyarginines comprise over five residues of l-arginine efficiently penetrate the cell membrane, and in some instances are part of more complex molecule which can deliver cargos to nucleus through nuclear localization signal (NLS). The general aim of this work is to reveal the properties of novel cell penetrating peptidomimetics which general structure is presented on figure 1. Such molecules contain several instances of 1-2,3-diaminopropionicacid (DAP) connected through the peptide bond and modified on its side chain amino groups by substitution of functionalized PEG moiety. For group of compounds we proposed the abbreviation DAPEG that is merged DAP and PEG. Obtained peptidomimetic which is labelled by 5/6- carboxyfluorescein moiety efficiently penetrates cell membrane and is transported directly to nucleus of primary and transformed cell without significant toxicity.

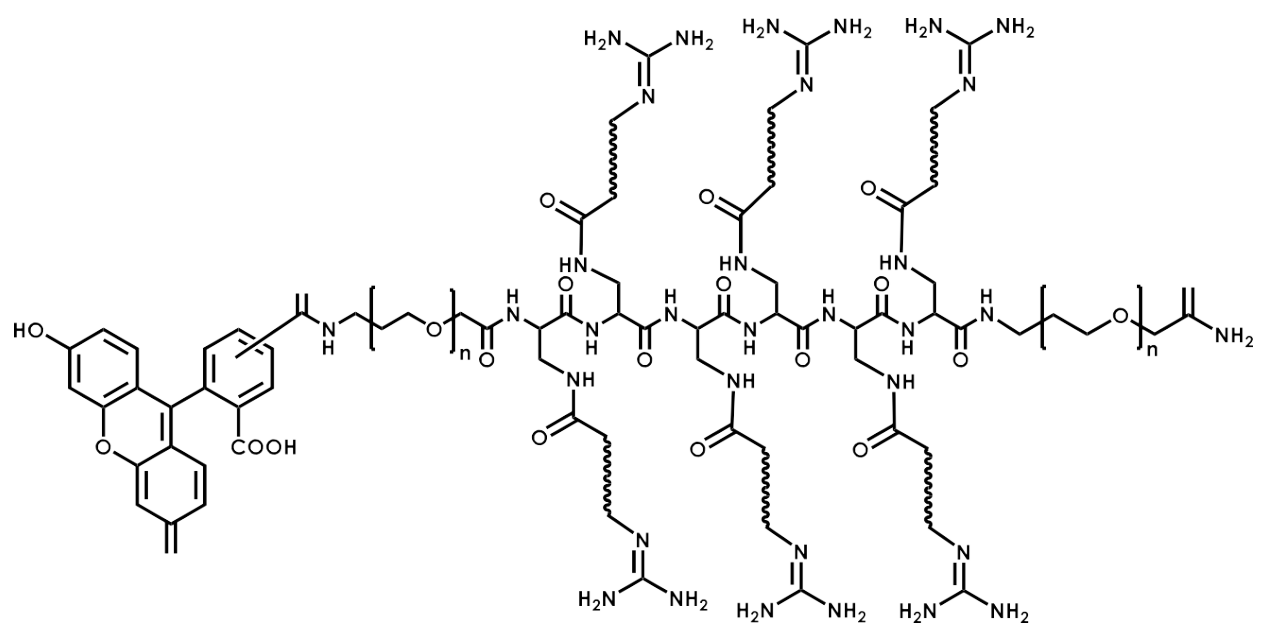

Figure 1: General formula of DAPEG mimetics.

Very recently we have synthesized a set of peptides and peptidomimetics that mimics hexaarginine one of the commoncell penetrating peptide standard. All compoundswere labelled by 5/6-carboxyfluorescein or rhodamine as cargo. Incubation of individual compounds with breast cell lines: HB2 and MDA-MB-231 for 2 hours at concertation of $10 \mu \mathrm{M}$ point out that some of the compoundsefficiently crossed the cell membrane(see figure 2 ).

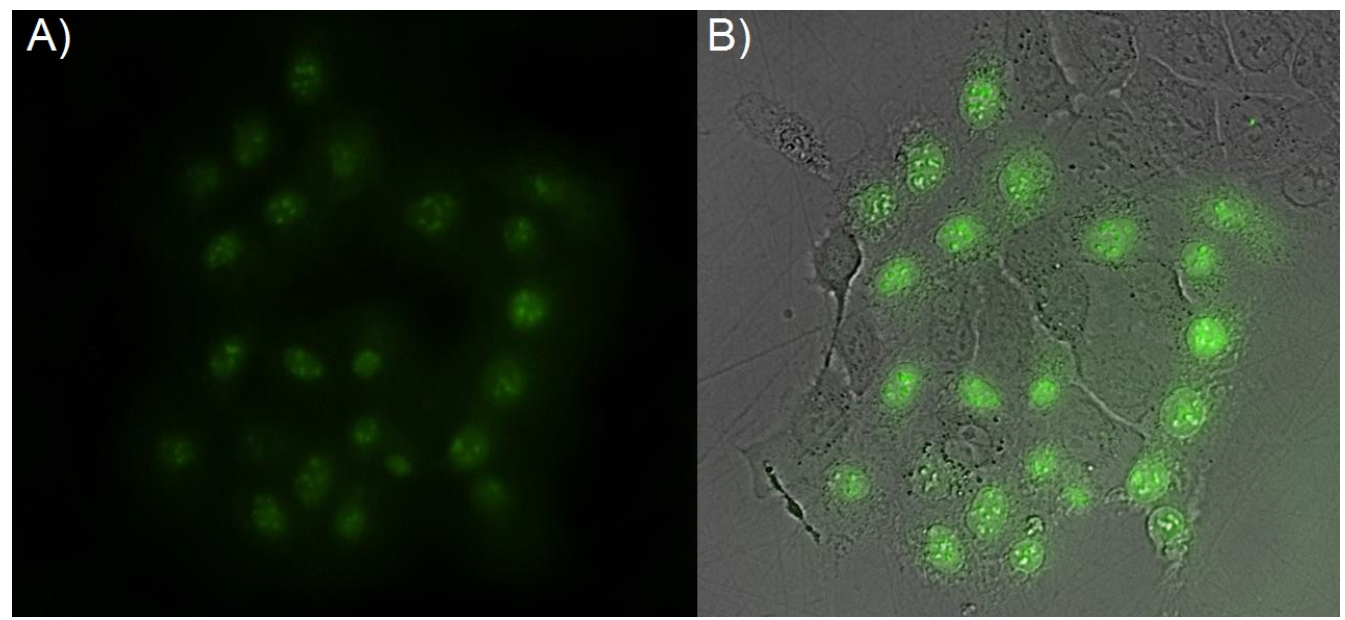

Figure 2: HB2 cell line incubated for 2 hours with $10 \mu M$ concentration of DAPEG compound. A) FITC fluorescence B) overlay of bright view and FITC fluorescence. 
For evaluation DNA - DAPEG interaction we employ EMSA gels that results are presented on figure 3. The selected compound was incubated with DNA short linear fragment (76 bp) at different concentration for 1 hour. After that time, the $20 \mu \mathrm{l}$ of DNA-DAPEG mixture was subjected to $2 \%$ gel supplemented with Sybr Safe View reagent. The gels were run for 45 minutes and further inspected under UV transilluminator

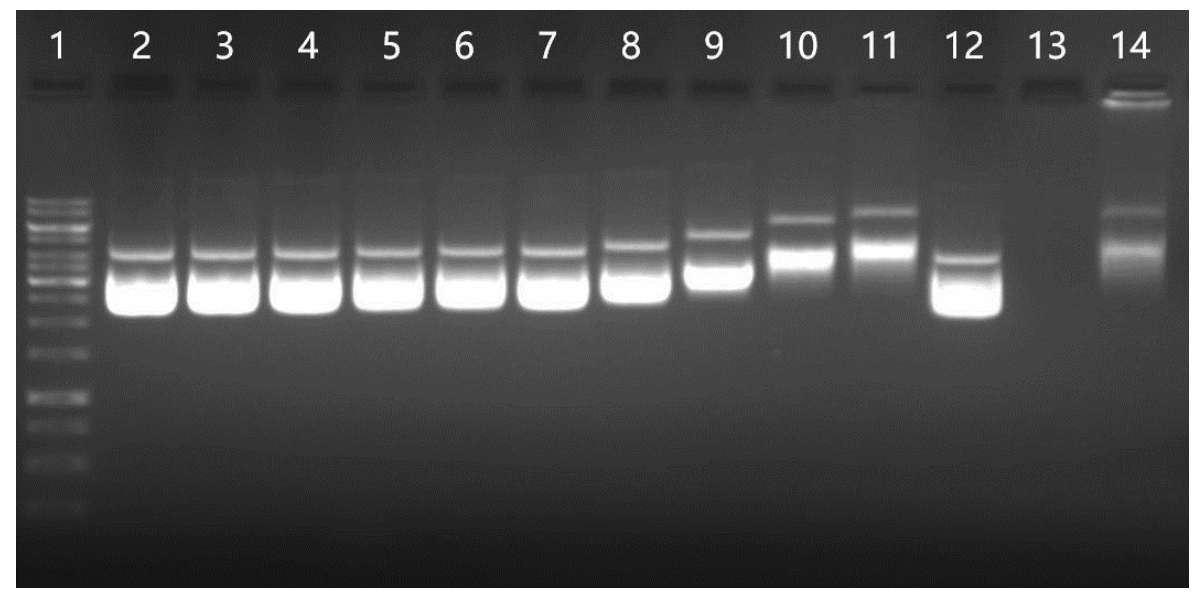

Figure 3: For evaluation DNA - DAPEG interaction we employ EMSA gels that results are presented on figure 3. The selected compound was incubated with DNA short linear fragment (76 bp) at different concentration for 1 hour. After that time, the $20 \mu \mathrm{l}$ of DNA-DAPEG mixture was subjected to 2\% gel supplemented with Sybr Safe View reagent. The gels were run for 45 minutes and further inspected under UV transilluminator.

\section{Conclusions}

We have synthesized the peptidomimetic that are rapidly cross the cell membrane and targets nucleus. This peptidomimetic display no significant cytotoxicity up to $50 \mu \mathrm{g} / \mathrm{m}$ and is able to bind to dsDNA in nanomolar concentration.

\section{Acknowledgements}

This work was supported by National Science Center Poland under grant UMO-2017/27/B/ST5/02061 (MW). 